\title{
Oxygen Octahedral Picker: A Digital Micrograph Script Tool for Extracting Quantitative Information From HAADF and ABF Images
}

\author{
Yi Wang ${ }^{1}$, Ute Salzberger ${ }^{1}$, Wilfried Sigle ${ }^{1}$, Y.Eren Suyolcu ${ }^{1}$ and Peter A. van Aken ${ }^{1}$ \\ 1. Stuttgart Center for Electron Microscopy, Max Planck Institute for Solid State Research, \\ Heisenbergstr. 1, 70569 Stuttgart, Germany
}

The multifaceted magnetic, electrical, and structural functionalities of perovskite oxides are underpinned by the distortions of the crystal lattice [1]. These distortions include the displacement of cations, deformation of oxygen octahedra $\left(\mathrm{BO}_{6}\right.$, where $\mathrm{B}$ is a transition metal atom), and collective tilts of the octahedral network. Controlling and engineering these distortions in the constituent oxides are crucial in designing and fabricating hetero-structures with novel functional properties that are absent in the bulk form. Atomistic understanding of these distortions and elucidation of their influence on the final properties requires imaging and measuring of atomic positions of both cations and oxygen. With the application of spherical aberration $\left(C_{\mathrm{s}}\right)$ correctors, sub-Angstrom atomic resolution is nowadays regularly achievable in both TEM and STEM. The recent application of the annular bright-field (ABF) imaging technique in perovskite oxides has become increasingly popular, as it enables simultaneous imaging of heavy and light elements and allows for simultaneous acquisition of other signals [2, 3]. Quantitative analysis of atom-column positions in high-resolution STEM images helps us gain new insight into materials behavior and address material problems. However, there are rare resources about methods for indexing atomic column positions in atomic resolution images, especially for the case of ABF images, as well as for the measurement accuracy.

In this contribution, we report the development of a software tool, written in Digital Micrograph scripting language [4], to extract quantitative information of the crystal lattice and of oxygen octahedron distortions of perovskite oxides from high-angle annular dark-field (HAADF) and ABF STEM images. The program is composed of two scripts: "B atom navigator" and "oxygen octahedral picker" ("O-O picker"), enabling analysis of the simultaneously acquired HAADF and ABF images. The second script uses the B atomic column positions obtained from the first script to detect surrounding oxygen atomic columns and to map all the octahedra on the ABF image. Center-of-mass and two-dimensional (2D) Gaussian fitting methods are implemented to locate positions of individual atom columns. The final results provided by this software are the coordinates of detected atomic columns, the atomic column intensities, the distances between the atomic columns, and the angles between selected atomic columns, based on the selection of the user. The flow chart of the script and the user interface of "O-O picker" script are shown in Fig. 1

The precision of atomic column distance measurements are evaluated on both simulated and experimental images. We demonstrate that sub picometer precision can be achieved on simulated STEM images. Experimentally, under a daily reproducible working condition (sample drift and slight sample contamination present), we can achieve 3 picometer and 4 picometer precisions for HAADF and ABF images. Figure 2 shows the details of the measurement precision test.

Finally, using practical examples, we demonstrate that the program works well for different orientations of the perovskite structure as well as for heterostructure interfaces. [5] 


\section{References:}

[1] R H Mitchell "Perovskites: Modern and Ancient", (Almaz, Thunder Bay)

[2] S D Findlay et al., Appl.Phys.Lett. 95 (2009), p.191913.

[3] E Okunishi et al., Microsc.Microanal.164 (2009), p.15.

[4] D R G Mitchell, B Schaffer, Ultramicroscopy 103 (2005), p.319.

[5] The research leading to these results has received funding from the European Union Seventh

Framework Program under Grant Agreement 312483-ESTEEM2 (Integrated Infrastructure Initiative I3).

(a)

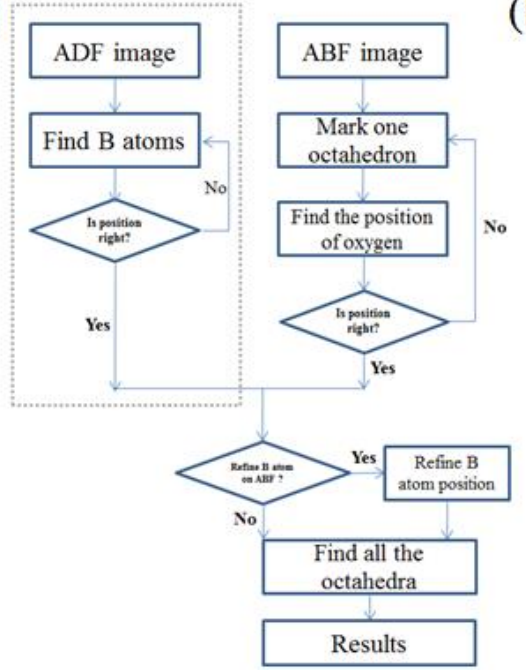

(b)

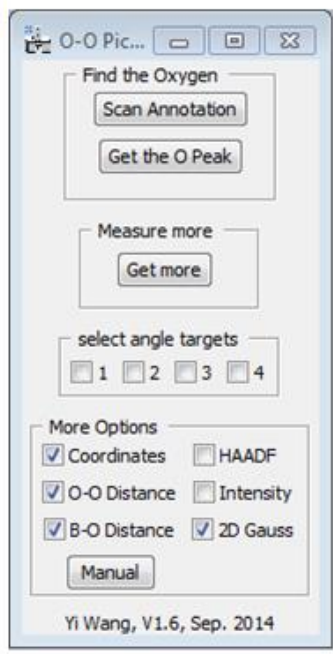

Figure 1. (a) Flow chart of the script. (b)The user interface of the oxygen octahedral picker script.

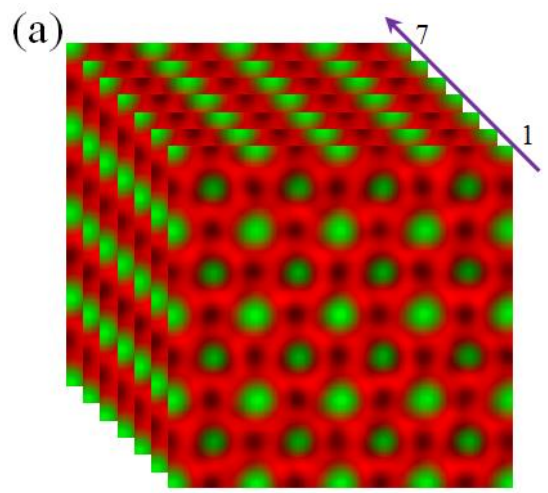

Image series: 7 frames

$2 \mu$ s per pixel (b)

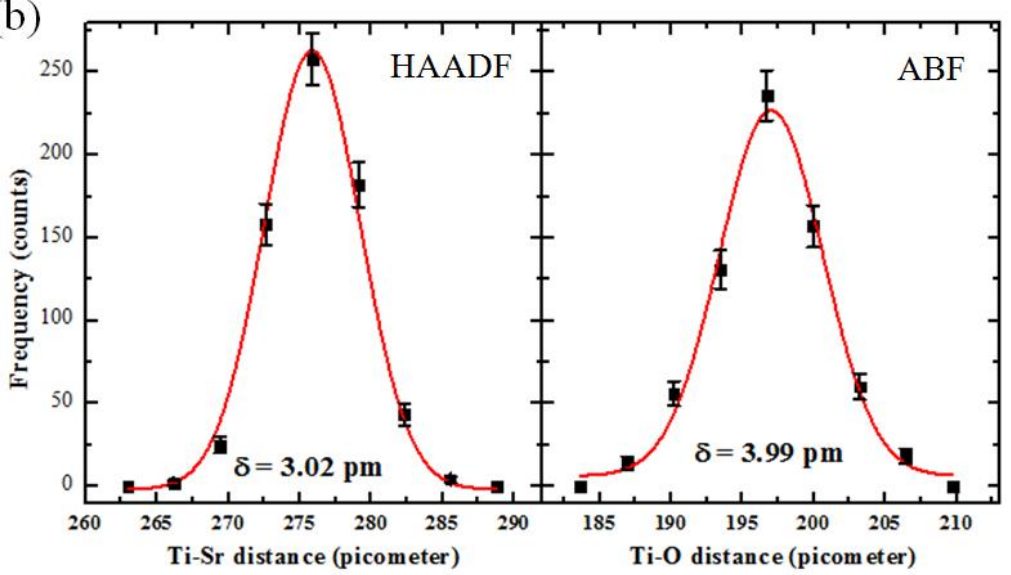

Figure 2. (a) Overlay of simultaneously acquired HAADF (green) and $\mathrm{ABF}$ (red) images of $\mathrm{SrTiO}_{3 .} 7$ frames were acquired with a short dwell time ( $2 \mu$ s per pixel). (b) Histograms of the measured $\mathrm{Ti}-\mathrm{Sr}$ and $\mathrm{Ti}-\mathrm{O}$ atomic column distances for HAADF and ABF images. 